\title{
The ExaVolt Antenna: Concept and Development Updates
}

\author{
Carl Pfendner ${ }^{1, \star}$ for the EVA collaboration \\ ${ }^{1}$ Dept. of Physics and CCAPP, The Ohio State University, 191 W. Woodruff Ave., Columbus, OH 43210, \\ USA
}

\begin{abstract}
A flux of ultrahigh energy neutrinos is expected both directly from sources and from interactions between ultrahigh energy cosmic rays and the cosmic microwave background. Using the cost-effective radio Cherenkov technique to search for these neutrinos, the ExaVolt Antenna (EVA) is a mission concept that aims to build on the capabilities of earlier radio-based balloon-borne neutrino detectors and increase the sensitivity to lower energies and fluxes. The novel EVA design exploits the surface of the balloon to provide a focusing reflector that aims to provide a signal gain of $\sim 30 \mathrm{dBi}$ (compared to $10 \mathrm{dBi}$ on ANITA). This increase in gain when combined with a large instantaneous viewing angle will yield a 10-fold increase in sensitivity and will allow this balloon-borne experiment to probe the expected low neutrino fluxes even at energies greater than $10^{19} \mathrm{eV}$. This contribution will present an overview of the mission concept, recent technology developments, and the results of a hang test of a 1:20-scale model which demonstrates the effectiveness of the design.
\end{abstract}

\section{Introduction}

A flux of ultra-high energy (UHE) neutrinos is expected due to interactions between UHE cosmic rays and the cosmic microwave background, as predicted by Berezinsky and Zatsepin [1]. Several experiments are currently pursuing the detection of the cosmogenic UHE neutrino flux using radio detection techniques. Detectors embedded within polar ice caps either several kilometers deep [2, 3] or at the surface [4] exploit the proximity to neutrino interactions in a large target volume. The ANITA [5] balloon-borne interferometer synoptically scans a significantly larger ice volume $\left(\sim\right.$ million $\left.\mathrm{km}^{3}\right)$ providing high sensitivity to the high energy end of the expected neutrino spectra.

An experiment's sensitivity to UHE-induced radio emissions is determined by the combination of visible target volume and live-time. Ground-based arrays implement large numbers of high duty cycle stations (37 for ARA and 1000 for ARIANNA) but the visible volume is limited by the refractive and attennuative properties of the ice. A balloon-borne detector, on the other hand, has a large visible target volume with a limited operational duty cycle. For balloon-borne detectors, the only practical improvement is to increase the antenna gain. The ExaVolt Antenna (EVA) [6] is a mission concept that builds on the success of three ANITA flights. EVA aims to implement a $\sim 30 \mathrm{dBi}$ gain antenna, exploiting the surface of a super-pressure balloon (SPB) to improve sensitivity to neutrino-induced radio-impulsive transients by a factor of 100 over ANITA.

^e-mail: pfendner.1@osu.edu 


\section{ExaVolt Antenna Design Concept}

The proposed EVA design will be the largest-aperture radio telescope ever flown on a balloon payload and will improve the sensitivity to neutrino-induced impulsive radio-frequency (RF) signals by a factor 100 or more over any previous experiment. This large improvement in gain can be obtained by using a portion of the balloon surface itself as part of the radio detector with a toroidal geometry as indicated in Fig. (1), where an RF-reflective film is applied to a section of the balloon surface 10-m high about the equator. An incoming plane wave impulse enters the balloon below the lower reflector rim opposite the active focusing area that will apply for that direction. Once reaching the opposite side of the balloon reflector surface, the incoming plane wave impulse is focused onto a focal plane inside the balloon. A set of patch feed antennas will be positioned at the focal plane of the reflector to receive the focused plane wave.

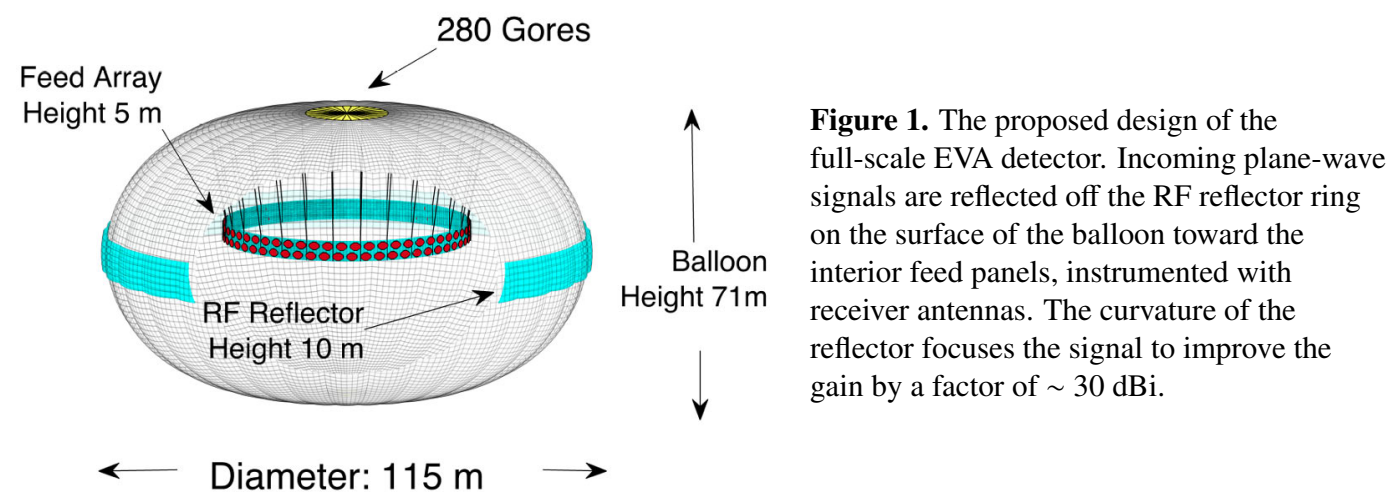

\section{Scale Model Hang Test}

In order to test the feasibility of the concept, the EVA collaboration performed a hang test of a 5.7m-diameter SPB constructed to our specifications by Aerostar International. The test was performed at NASA Wallops Flight Facility in September 2014. This balloon included 50-cm high aluminizedmylar reflective panels attached to the balloon at the equator by the vendor using polyethylene adhesive tape. A view of the fully-deployed balloon during the test is shown in Fig. (2).

Prior to inflation of the balloon, we inserted a scale-model of the antenna feed array membrane through the top end-plate of the balloon. In order to accommodate the insertion, the feed array was folded and was attached to internal support lines which were used to expand the array from its folded state during inflation and which provided support for the array upon final deployment. The feed array membrane was instrumented with dual polarization sinuous patch antennas over a portion of its circumference, and four of these antennas were also instrumented with microwave receivers coupled to RF-over-fiber transceivers which inserted the RF signals into optical fiber, as shown in Fig. (2).

The two primary goals of the test were to demonstrate (1) that it was possible to deploy a feed membrane antenna array within a super-pressure balloon, and (2) that the response of the balloon and patch antennas would function as predicted by antenna models when subject to an external impulsive plane-wave signal. To create this external signal, we used a 2.6 meter offset parabolic dish as an RF collimator, fed by a broadband dual-ridge horn emitting microwave impulses. We made several compromises on the scalability for the sake of the test: the number of gores was set at 28 for this 

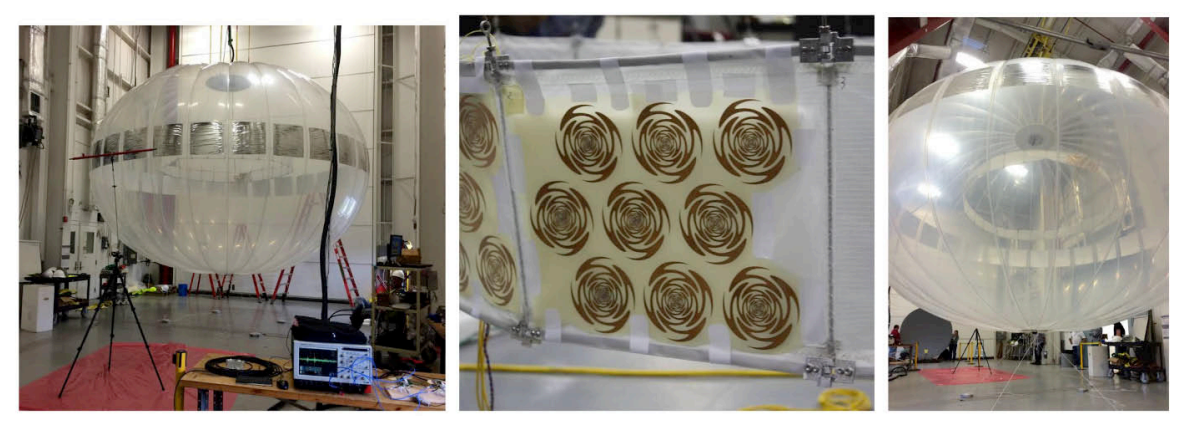

Figure 2. Left: The fully inflated hang test balloon. Photogrammetry was used to determine the geometry of the ballon and reflector after inflation. Center: One panel of the feed array. Four of these sinuous patch antennas were instrumented with electronics. Right: The hang test setup with the test signal emitter dish shown in the background.

balloon, rather than 280, to keep construction simple; and the width of the collimation dish was much smaller than the receiving width of the reflective panels due to constraints of portability of the dish. These compromises were understood and incorporated in the simulations used for comparison. The results of the microwave test and the simulations are summarized in Fig. (3). It is evident that the temporal coherence of the arriving impulse is preserved in the focused pulse. The observed pulse gain matches the model estimates within about $2 \mathrm{~dB}$, a solid validation of the simulation tools and our methodology. This agreement serves as additional confirmation of the feasibility of the concept as a method to improve signal gain.
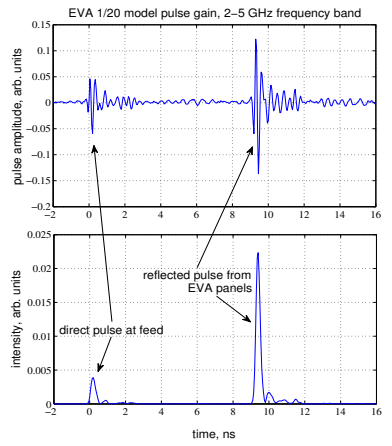
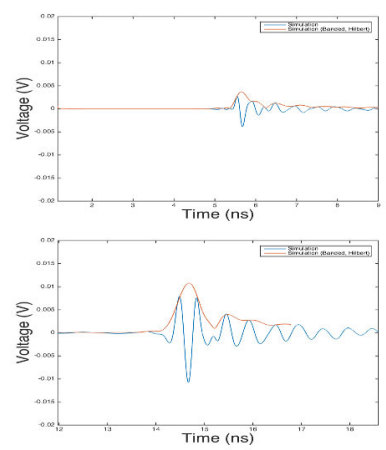

Figure 3. On the left is shown the incident and reflected pulse as measured during the hang test. The upper plot shows the recorded voltage and the lower shows the intensity. Shown on the right is the voltage for the incident (top) and reflected (bottom) pulse as predicted by the simulation program XFdtd [7] for the hang test geometry.

\section{Full-scale Simulation and Prediction for the Future}

In order to predict the response of the full-scale EVA detector and to investigate further possibilities of design improvements, we have created detailed antenna models for the super pressure balloon geometry using the currently available $18.7 \mathrm{Mcft}$ balloon with a radius of $\sim 57 \mathrm{~m}$. Using this model, we have investigated the response of the reflection geometry with three electromagnetic simulation packages. The packages used are as follows: Numerical Electromagnetic Code v.4 (NEC4) [8] which uses the method of moments; General Reflector Antenna Simulation Program (GRASP) [9] using 
physical optics; and XFdtd v.7 [7] which is based on the finite-difference time-domain method. Using each of these methods independently, we have found that simulations using low-gain feed antennas predict gains of 23-27 dBi from the reflector ring in the UHF band. Results from one of these tests is shown in Fig. (4).

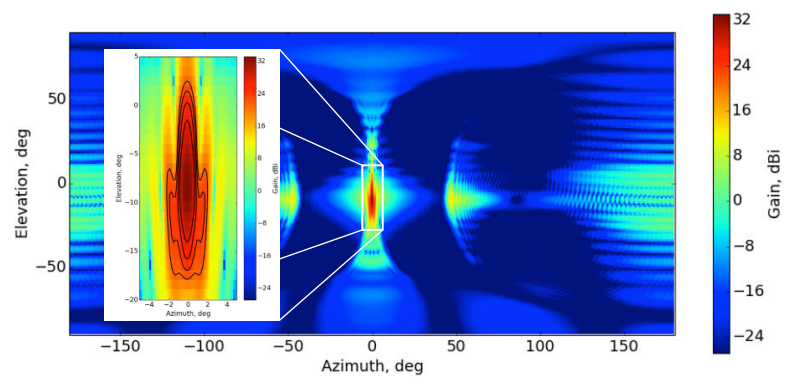

Figure 4. GRASP model power pattern for a 18.7 Mcft EVA model, using a feed with a response matched to the reflector. By using feeds matched to the antenna response of the reflector in the GRASP simualtions, the predictions of the gain can be improved up to $32 \mathrm{dBi}$ as shown here.

The results of the hang test confirm both the effectiveness of the concept of using the balloon as a focusing reflector and of the agreement of the measured data with expectations from simulation. Using the expected gain in sensitivity from these results, one can predict the improvement in sensitivity to neutrino-induced RF signals and thus to the neutrino flux itself. This flux sensitivity is shown in Fig. (5) and predicts that the EVA concept will be a competitive and valuable contributor to the detection of UHE neutrinos.

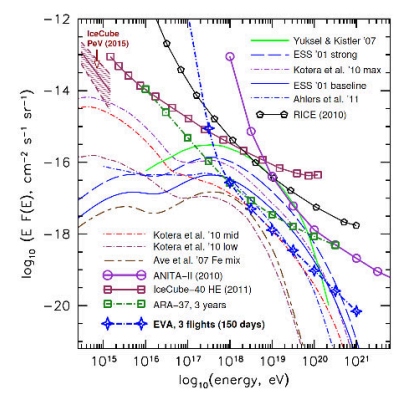

Figure 5. Estimated flux limits for a full-scale EVA detector after 150 days of flight time. Also shown are estimated sensitivity curves for other UHE neutrino detection experiments and several flux models.

\section{References}

[1] V. S. Berezinsky and G. T. Zatsepin, Phys. Lett., B28, 423 (1969); V. S. Berezinsky and G. T. Zatsepin, Sov. J. Nucl. Phys., 11, 111 (1970)

[2] M. G. Aartsen et al., Phys.Rev., D88, 112008, (2013); M. G. Aartsen et al., Science, 6161, 342, 1242856 (2013).

[3] P. Allison et al., Astropart.Phys., 35, 457 (2012).

[4] S. R. Klein at al., IEEE Trans. Nucl. Sci., 60637 (2013).

[5] P.W. Gorham et al., Phys. Rev., D82, 022004 (2010); P.W. Gorham et al., Erratum (2010).

[6] P.W. Gorham et al., Astropart. Phys., 35242 (2011).

[7] http://www.remcom.com/xf7

[8] G. J. Burke, E. K. Miller, and A. J. Poggio, IEEE Intl Symp. National Radio Science (URSI) (2004).

[9] http://www.ticra.com/products/software/grasp 